\title{
Photoproduction of Upsilon states in ultraperipheral collisions at the LHC
}

\author{
Magno Machado ${ }^{1, \star}$ \\ ${ }^{1}$ High Energy Physics Phenomenology Group, GFPAE IF-UFRGS \\ Caixa Postal 15051, CEP 91501-970, Porto Alegre, RS, Brazil
}

\begin{abstract}
The exclusive photoproduction of upsilon states is investigated in the context of ultra-peripheral collisions at the LHC energies. Predictions are presented for their production in proton-proton, proton-nucleus and nucleus-nucleus collisions at the energies available at the LHC run 2. The theoretical framework considered in the analysis is the light-cone color dipole formalism, which includes consistently parton saturation effects and nuclear shadowing corrections. For $\mathrm{PbPb}$ collisions we consider both the coherent and incoherent contributions.
\end{abstract}

\section{Introduction}

The exclusive photoproduction of heavy vector mesons is a kind of diffractive process where, besides a soft scale characterized by the hadron size, there is clearly a hard scale (mesons mass $m_{V}$ ) that allows to analyse the reaction from the perturbative QCD point of view. This advantage creates ways to investigate the pomeron exchange which could lead to a better understanding of this object in terms of QCD. Its investigation in ultraperipheral collisions (UPCs) [1] is an essential tool to understand the low-x physics and also to investigate the gluon density in this regime. In the UPC case, the exclusive photoproduction dominates the process through the emission of quasi-virtual photons which interact with the target. The theoretical approaches addressing such reactions include vector meson dominance (VDM), perturbative QCD calculations, $k_{T}$-factorization approach and color dipole formalism. The photon-target interaction amplitude, when considering the light-cone dipole formalism [2], can be written as a convolution between the photon-meson wave functions overlap and the elementary dipoletarget cross section [3]. In addition, the process considered here is quasi-elastic, $Q^{2} \approx 0$, and in the region of small-x, the gluon density may increase to the point where gluon fusion, $g \mathrm{~g} \rightarrow \mathrm{g}$, becomes significant. This kind of fusion produces nonlinear effects in the evolution equations. For instance, at mid-rapidity the typical value of Bjorken variable is $x=\frac{m_{r}}{\sqrt{s_{A A}}} \simeq 10^{-3}$ for $\mathrm{PbPb}$ collisions at the LHC. Differently from DGLAP equations which is a linear equation, dipole models incorporate linear and non-linear effects[4]. In pQCD the exclusive meson photoproduction has a differential cross-section $\propto\left[x g\left(x, Q^{2}\right)\right]^{2}$, where the gluon distribution functions do not take into account the effects of saturation since they are evolved by DGLAP equations. The inclusion of parton saturation and nuclear effects are crucial in describing even the experimental observations in heavy-ion collisions at RHIC (for instance, see Ref. [5]). Within the color dipole approach one can introduce information on dynamics

^e-mail: magnus@if.ufrgs.br 
beyond the leading logarithmic QCD approach for meson production and computing predictions for the radially excited states is a reasonably easy task [3]. From the experimental point of view, the considered process is quite clear due to the presence of two rapidity gaps. The rapidity gap describes a region between the axis beam and the decay of meson $\left(e^{+}+e^{-}, \mu^{-}+\mu^{+}\right)$free of particles. In this way, the experimental observation of this kind of quasi-diffractive process is facilitated. In addition, the absorption corrections in this case is not strong as in the corresponding final state in proton-proton collisions.

In this contribution we summarize the results obtained in Refs. [6, 7], where we investigate the exclusive production of $\Upsilon(1 S)$ and its radially excited states $\Upsilon(2 S)$ and $\Upsilon(3 S)$ in PbPb collisions for LHC energy. In Ref. [7] the coherent photoproduction of $\Upsilon$ states at various energies in $p p, \mathrm{pPb}$ $\mathrm{PbPb}$ collisions at the $\mathrm{LHC}$ has been considered. Those calculations were carried out in the theoretical framework of the color light-cone dipole formalism [2] and focused only on the coherent channel where the initial state particles remain intact after interaction. It was shown that the corresponding predictions describe correctly the experimental results from LHCb Collaboration [8] for $\Upsilon$ photoproduction in $p p$ collisions. Those data were obtained for typically large rapidities and the $x$-values to be covered are increasingly smaller for forward rapidities. We roughly get $x=\frac{m_{\Upsilon}}{\sqrt{S_{A A}}} e^{-y} \simeq 8 \times 10^{-5}$ at $y=3$ and it is clear that gluon dynamics is being probed at extremely low- $x$ and low perturbative scales $\mu^{2} \simeq 20 \mathrm{GeV}^{2}$. This kinematical range is in the limit of application of usual pQCD and saturation approach should be relevant. For nuclear targets, the nuclear saturation scale $Q_{s a t, A}^{2} \simeq c A^{1 / 3} Q_{s a t, p}^{2}$ (with $c \simeq 0.3$ ) reaches $2 \mathrm{GeV}^{2}$ in those cases [9]. In Ref. [6], a detailed study of the incoherent cross section for the upsilon states has been done. This is quite important, as it was pointed out that incoherent diffraction probes the fluctuations in the interactions strengths of multiparton Fock states in the nuclear wavefunctions [10]. The connection between incoherent diffraction and fluctuations is a quite rich subject and the pioneering works are found in Refs. [11]. Recently, the topic is very active and we call attention to the following works $[12,13]$.

This contribution is organized as follows. In the next section we give the main theoretical information to obtain the rapidity distribution of coherent and incoherent production of $\Upsilon(1 S, 2 S, 3 S)$ states in hadron-hadron and heavy ion collisions for the energies available at the LHC. The approach considered here is quite robust due to the successful description of experimental results measured by LHCb Collaboration [8] for $\Upsilon(1 S)$ in $p p$ collisions. In the section 3 we present the phenomenological calculations, discuss the main theoretical uncertainties and a comparison with other approaches is performed. For instance, we compare the present calculation to the predictions available using the STARlight Monte Carlo [14, 15]. Finally, we show the main conclusions.

\section{Theoretical Framework}

The exclusive meson photoproduction in nucleus-nucleus collisions can be factorized in terms of the equivalent flux of photons of the nucleus projectile and photon-target production cross section [1]. In UPCs there is the absence of strong interactions between the projectile particle and the target. In this case the reaction is characterized by impact parameter $>2 R_{A}$ and as the interaction is ultrarelativistic and purely electromagnetic, one can use the Weizsäcker-Williams approximation [1]. The photon energy spectrum, $d N_{\gamma}^{A} / d \omega$, which depends on the photon energy $\omega$, is well known [1]. The rapidity distribution for $\Upsilon$ states photoproduction in $A A$ collisions can be written down as,

$$
\frac{d \sigma}{d y}(A A \rightarrow A \otimes \Upsilon(n S) \otimes Y)=\omega \frac{d N_{\gamma}^{A}}{d \omega} \sigma(\gamma A \rightarrow \Upsilon(n S) Y)+(y \rightarrow-y),
$$


where the photon flux in nucleus is denoted by $d N_{\gamma}^{A} / d \omega$ and $Y=A$ (coherent case) or $Y=A^{*}$ (incoherent case). The symbol $\otimes$ denotes the large rapidity gap between the produced meson and the final states nucleus.

Similar calculation can be carried out in proton-proton and proton-nucleus interactions. For highly energetic protons as those produced by the LHC beams the photon flux can be approximated by $[1,15]$

$$
\frac{d N_{\gamma}^{p}}{d \omega}=\frac{\alpha_{e m}}{2 \pi \omega}\left[1+\left(1-\frac{2 \omega}{\sqrt{s}}\right)^{2}\right]\left[\ln \Omega-\frac{11}{6}+\frac{3}{\Omega}-\frac{3}{2 \Omega^{2}}+\frac{1}{3 \Omega^{3}}\right]
$$

where $\Omega=1+0.71 \mathrm{GeV}^{2} / Q_{\min }^{2}$ e $Q_{\min }^{2}=\left(\omega / \gamma_{L}\right)^{2}$, with $\gamma_{L}=\sqrt{s} /\left(2 m_{p}\right)$. On the other hand, for nucleus having charge $Z$, the photon flux is approximately given by $[1,15]$

$$
\frac{d N_{\gamma}^{A}}{d \omega}=\frac{2 Z^{2} \alpha_{e m}}{\pi}\left[\xi K_{0}(\xi) K_{1}(\xi)-\frac{\xi^{2}}{2}\left(K_{1}^{2}(\xi)-K_{0}^{2}(\xi)\right)\right],
$$

where $\xi=\omega\left(R_{p}+R_{A}\right) / \gamma_{L}$ for $p A$ collisions and $\xi=\omega\left(2 R_{A}\right) / \gamma_{L}$ for $A A$ collisions. We will discuss about the rapidity distribution for those collisions in next section.

The produced state with mass $m_{V}$ has rapidity $y \simeq \ln \left(2 \omega / m_{V}\right)$ and the square of the $\gamma A$ centreof-mass energy is given by $W_{\gamma A}^{2} \simeq 2 \omega \sqrt{s}$. The photon-Pomeron interaction will be described within the light-cone dipole frame, where the probing projectile fluctuates into a quark-antiquark pair with transverse separation $r$ (and momentum fraction $z$ ) long after the interaction, which then scatters off the hadron. The cross section for exclusive photoproduction of $\Upsilon$ states off a nucleon target is given by,

$$
\sigma(\gamma p \rightarrow \Upsilon p)=\frac{\left|\sum_{h, \bar{h}} \int d z d^{2} r \Psi_{h, \bar{h}}^{\gamma} \sigma_{d i p}(x, r) \Psi_{h, \bar{h}}^{V_{*}^{*}}\right|^{2}}{16 \pi B_{\Upsilon}},
$$

where $\Psi^{\gamma}$ and $\Psi^{V}$ are the light-cone wavefunction of the photon and of the vector meson $(V=\Upsilon)$, respectively. The dipole-proton cross section is denoted by $\sigma_{d i p}(x, r)$ and the diffractive slope parameter by $B_{V}$. In this context, we are implicitly assuming that the proton shape is Gaussian and that the impact parameter dependence factorizes out from the dipole-nucleon scattering amplitude. Here, we consider the energy dependence of the slope using the Regge motivated expression [7].

The exclusive photoproduction off nuclei for coherent and incoherent processes can be simply computed in high energies where the large coherence length $l_{c} \gg R_{A}$ is fairly valid. The expressions for both cases are given by [16],

$$
\begin{aligned}
\sigma(\gamma A \rightarrow \Upsilon A) & =\int d^{2} b\left|\sum_{h, \bar{h}} \int d z d^{2} r \Psi_{h, \bar{h}}^{\gamma} \Psi_{h, \bar{h}}^{V *}\left[1-\exp \left(-\frac{1}{2} \sigma_{d i p}(x, r) T_{A}(b)\right)\right]\right|^{2}, \\
\sigma\left(\gamma A \rightarrow \Upsilon A^{*}\right) & =\int d^{2} b \frac{T_{A}(b)}{16 \pi B_{V}}\left|\sum_{h, \bar{h}} \int d z d^{2} r \Psi_{h, \bar{h}}^{\gamma} \Psi_{h, \bar{h}}^{V *} \sigma_{d i p}(x, r) \exp \left[-\frac{1}{2} \sigma_{d i p}(x, r) T_{A}(b)\right]\right|^{2},
\end{aligned}
$$

where $T_{A}(b)=\int d z \rho_{A}(b, z)$ is the nuclear thickness function. In the numerical evaluations, we have considered the boosted Gaussian wavefunction and several phenomenological saturation models, which encode the main properties of the saturation approaches. Accordingly, the cross sections above include both the skwedness and real part of amplitude corrections. Namely, we multiply the result 
above by $K^{2}=R_{g}^{2}\left(1+\beta^{2}\right)$, where $\beta=\tan \left(\pi \lambda_{e f} / 2\right)$ is the ratio of real to imaginary parts of the scattering amplitude and $R_{g}$ incorporates the off-forward correction (see [7] for details). The effective power on energy, $\lambda_{e f}$ is determined for each case. In order to take into account the threshold correction for the dipole cross section, we have multiplied them by a factor $(1-x)^{7}$.

Finally, we set the parameters and phenomenological models to be considered in next section. For the slope parameter it was considered the energy dependency from the Regge phenomenology [7],

$$
B_{\Upsilon}=b_{e l}^{\Upsilon}+2 \alpha^{\prime} \log \left(\frac{W_{\gamma A}^{2}}{W_{0}^{2}}\right)
$$

with $\alpha^{\prime}=0.164 \mathrm{GeV}^{-2}, W_{0}=95 \mathrm{GeV}, b_{e l}^{\Upsilon_{(1 S)}}=3.68 \mathrm{GeV}^{-2}, b_{e l}^{\Upsilon_{(2 S)}}=3.61 \mathrm{GeV}^{-2}$ and $b_{e l}^{\Upsilon_{(3 S)}}=3.57$ $\mathrm{GeV}^{-2}$. It will be taken into account only for the incoherent cross section. For the meson wavefuntion, we will use the Boosted-Gaussian model [17] because it can be applied in a systematic way for excited states. The corresponding function is given by [18],

$$
\phi_{n S}(r, z)=\left[\sum_{k=0}^{n-1} \alpha_{n S, k} R_{n S}^{2} \hat{D}^{2 k}(r, z)\right] G_{n S}(r, z),
$$

with $\alpha_{n S, 0}=1$. The operator $\hat{D}^{2}(r, z)$ is defined by

$$
\hat{D}^{2}(r, z)=\frac{m_{f}^{2}-\left(\frac{1}{r} \partial_{r}+\partial_{r}^{2}\right)}{4 z(1-z)}-m_{f}^{2}
$$

and it acts on the following generatrix function

$$
G_{n S}(r, z)=\mathcal{N}_{n S} z(1-z) \exp \left(-\frac{m_{f}^{2} \mathcal{R}_{n S}^{2}}{8 z(1-z)}-\frac{2 z(1-z) r^{2}}{\mathcal{R}_{n S}^{2}}+\frac{m_{f}^{2} \mathcal{R}_{n S}^{2}}{2}\right) .
$$

The main physical quantity is the dipole scattering cross section. We consider the following phenomenological models in our analysis: GBW [19], CGC [20] and BCGC [21]. The GBW model is defined by the eikonal shape for the dipole cross section,

$$
\sigma_{q \bar{q}}^{G B W}(x, r)=\sigma_{0}\left(1-e^{-r^{2} Q_{s}^{2}(x) / 4}\right)
$$

where $\sigma_{0}=2 \pi R^{2}$ is a constant and $Q_{s}^{2}(x)=\left(x_{0} / x\right)^{\lambda} \mathrm{GeV}^{2}$ denotes the saturation scale. We also consider the CGC model [20], based in the Color Glass Condensate framework, in which gluon saturation effects are incorporated via an approximate solution of the Balitsky-Kovchegov equation [4]. The expression for the CGC model is given by,

$$
\sigma_{q \bar{q}}^{C G C}(x, r)=\sigma_{0} \begin{cases}\mathcal{N}_{0}\left(\frac{r Q_{s}}{2}\right)^{\gamma_{e f f}(x, r)} & : r Q_{s} \leq 2 \\ 1-e^{-A \ln ^{2}\left(B r Q_{s}\right)} & : r Q_{s}>2\end{cases}
$$

where $\gamma_{e f f}(x, r)=2\left(\gamma_{s}+(1 / \kappa \lambda \ln (1 / x)) \ln \left(2 / r Q_{s}\right)\right)$ is the effective anomalous dimension and one has the constant $\kappa=9.9$.

In order to investigate the theoretical uncertainty associated to the models for the dipole cross section, we use the original values (OLD label) of parameters for the fits including the charm contribution. That is, for GBW-OLD we follow Ref. [19], for CGC-OLD Ref. [22] is considered and bCGC-OLD refers to Ref. [21]. The bCGC model uses the same functional form of Eq. (10) and replaces the saturation scale in the following way: $Q_{s}^{2}(x) \rightarrow Q_{s}(x, b)^{2}=\left(x_{0} / x\right)^{\lambda} \exp \left[-b^{2} /\left(2 \gamma_{s} B_{C G C}\right)\right]$. Moreover, we consider the updated version of those models, GBW-NEW [13], CGC-NEW [23] and 


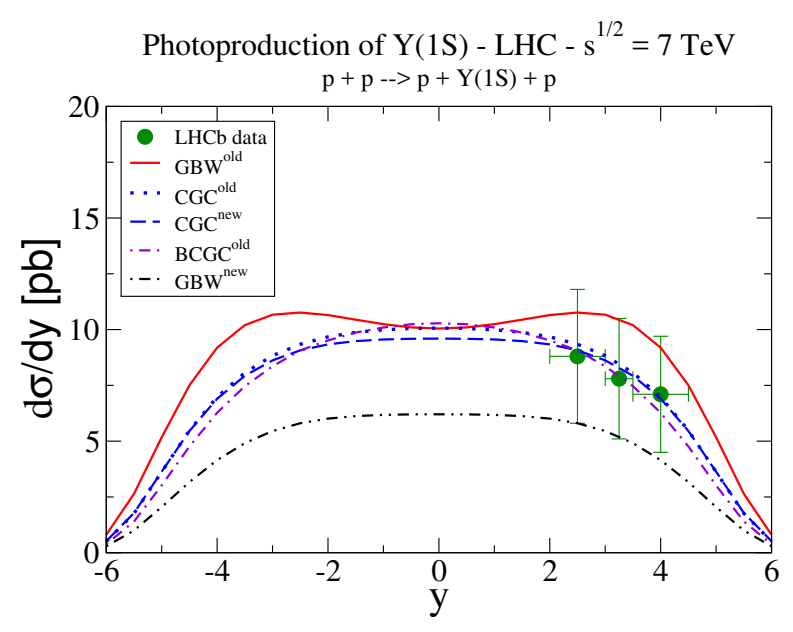

Figure 1. Rapidity distribution for the $\Upsilon(1 S)$ state in $p p$ collisions at $\sqrt{s}=7 \mathrm{TeV}$ using boosted gaussian model (BG) for wavefunction. Theoretical uncertainty here comes from distinct models for dipole cross section. Data from LHCb Collaboration[8]

bCGC-NEW [23], respectively. A comment is in order here: the GBW-NEW parametrization is very different from other color dipole fits, as it includes energy evolution of the subnucleonic shape of the proton and it can potentially significantly affect the incoherent cross section. In particular, GBW-OLD and GBW-NEW are qualitatively very different and GBW-NEW was not fitted to all $F_{2}$ small- $x$ data (DESY-HERA) as discussed in details in Ref. [13].

\section{Results and discussions}

Let us start the analysis by computing the theoretical predictions for the proton-proton case. In $p p$ collisions, the rapidity distribution of vector meson $V$ is given by

$$
\frac{d \sigma}{d y}(p+p \rightarrow p+p+V)=S_{g a p}^{2}\left[\omega \frac{d N_{\gamma}^{p}(\omega)}{d \omega} \sigma(\gamma p \rightarrow V+p)+(y \rightarrow-y)\right],
$$

where the rapidity of the produced meson is related to the photon energy by $y \simeq \ln \left(2 \omega / m_{V}\right)$. The parameter $S_{\text {gap }}^{2}$ quantifies the absorptive corrections[24], and in such a process one has the presence of large rapidity gap between the produced meson and the final state protons. In the present calculation we will take $S_{\text {gap }}^{2} \approx 0.8-0.9$. Finally, the notation $(y \rightarrow-y)$ indicates the symmetry target-projectile in the $p p$ collision. In Figure 1, it is presented the results for photoproduction $\Upsilon(1 S)$ in $p p$ collisions considering the different models presented in the last section. The relative normalization and the overall behavior on rapidity are fairly reproduced by all the models in the forward region in comparison to the experimental results from LHCb Collaboration [8]. Given the present level of the experimental uncertainties it is not possible to make definitive statements about the precision of the distinct models investigated. The theoretical uncertainty reaches a factor two considering the same wave-function and distinct dipole cross sections. Predictions for the upsilon states in $p p$ collisions at $13 \mathrm{TeV}$ and $p A$ collisions at 8.2 TeV are presented in Ref. [7]. The general trend follows the one predicted at lower energy, including the theoretical uncertainty and overall behavior. The cross sections are quite 
sizeable and the relative contribution of the radial excited states compared to the lowest state follows the pattern shown at $y=0$. Namely, $\sigma[\Upsilon(2 S)] / \sigma[\Upsilon(1 S)] \simeq 0.17$ and $\sigma[\Upsilon(3 S)] / \sigma[\Upsilon(1 S)] \simeq 0.083$. Our predictions for the $\Upsilon$ state ratios are lower that those predicted by STARlight Monte Carlo, as presented in Ref. [14]. The origin can be the fact that the different states are obtained from an extrapolation of HERA-DATA and using a fixed ratio for the distinct states in [14]. In our case, the evolution on energy is dynamically generated by parton saturation approach models and the meson wavefuntions have non-trivial behavior on the overlap function.

The calculation discussed above can be directly compared to another theoretical approaches. For instance, the present result is consistent with the NLO pQCD analysis done in Ref. [25], which also compares the outcoming predictions to the $\mathrm{LHCb}$ data. It is verified that the theoretical uncertainty from color dipole approach is somewhat smaller than the presented there (particularly, LO and NLO pQCD predictions present large disparities). Moreover, it is worth to note that the present work updates the analysis done in Ref. [18], where the photoproduction of $\Upsilon$ states in $p p$ collisions has been done for the first time in the scope of color dipole approach, also used in [26] for $\Upsilon(1 S)$. The focus here is to provide predictions for the next (and current) runs of LHC.

In Ref. [7] predictions for upsilon states in $p A$ ultra-peripheral collisions were presented. In particular, in proton-lead collisions if the quarkonium rapidity, $y$, is positive in the nucleus beam direction its rapidity distribution reads as [1]:

$$
\frac{d \sigma}{d y}(P b+p \rightarrow P b+p+V)=\frac{d N_{\gamma}^{P b}(y)}{d \omega} \sigma_{\gamma p \rightarrow V+p}(y)+\frac{d N_{\gamma}^{p}(-y)}{d \omega} \sigma_{\gamma P b \rightarrow V+P b}(-y),
$$

where $\frac{d N_{\gamma}(y)}{d \omega}$ is the corresponding photon flux. The case for the inverse beam direction is straightforward. We use the Weiszäcker-Williams method to calculate the flux of photons from a charge $Z$ nucleus as referred in the previous section. In the numerical calculation we disregard the contribution coming from the photon flux related to the proton source. The integrated cross section (in the range $2 \leq y \leq 4.5$ ) is predicted to be $243.41 \pm 20.37 \mathrm{pb}$ and $4.97 \pm 0.48 \mathrm{pb}$, respectively. The error includes the theoretical uncertainty related to the model of the dipole cross section. When considering the higher energy of $\sqrt{s}=8.2 \mathrm{TeV}$ the values found are $340.51 \pm 36.76 \mathrm{pb}$ and $7.14 \pm 0.85 \mathrm{pb}$. Moreover, for the lowest state $\Upsilon(1 S)$ one has $2.92 \pm 1.43 \mathrm{pb}$ at $\sqrt{s}=5.02 \mathrm{TeV}$ and $5.45 \pm 2.04 \mathrm{pb}$ at $\sqrt{s}=8.2$ $\mathrm{TeV}$. In Figure 2 the rapidity distribution is shown for the $\Upsilon(1 S)$ state at $\sqrt{s}=8.2 \mathrm{TeV}$. In addition, we compare our results to recent CMS Collaboration data [27], where the theoretical predictions are consistent with measurements within the errors. The model deviation is more intense on the very forward rapidity and the theoretical uncertainty reaches a factor two similarly to the $p p$ case. The rapidity distribution exhibits directly the influence of the $x$-dependence of the dipole cross section in the interval $0 \leq y \leq 4$, and specially for rapidities around $y=3$. The shapes are similar for the lowest state and its radial excited states (see Ref. [7]). Therefore, it is feasible that a consideration of $\Upsilon$ photoproduction in this rapidity interval offers potential in discriminating models of dipole cross sections. It is timely having a measurement of $\Upsilon$ production at mid-rapidity, as the corresponding cross section in the $\psi$ case it is a challenge when considering the color dipole approaches. The predictions can be directly compared to the work in Ref. [30], where the perturbative two-gluon exchange formalism has been considered. We have not verified a second peak in the rapidity distribution as presented in [30]. The reason is that the dynamics embedded in the color dipole approach considered here corresponds to strong shadowing corrections. The ratio $\sigma[\Upsilon(2 S)] / \sigma[\Upsilon(1 S)]$ and $\sigma[\Upsilon(3 S)] / \sigma[\Upsilon(1 S)]$ are still of same order of magnitude compared to the proton-proton case.

Concerning the theoretical predictions for the coherent process for $\mathrm{PbPb}$ collisions at $5.5 \mathrm{TeV}$, we use the Glauber-Gribov approach presented in previous sectiona and disregard any absorptive corrections. In Figure 3, it is presented the results for photoproduction of $\Upsilon$ states, including its radial 

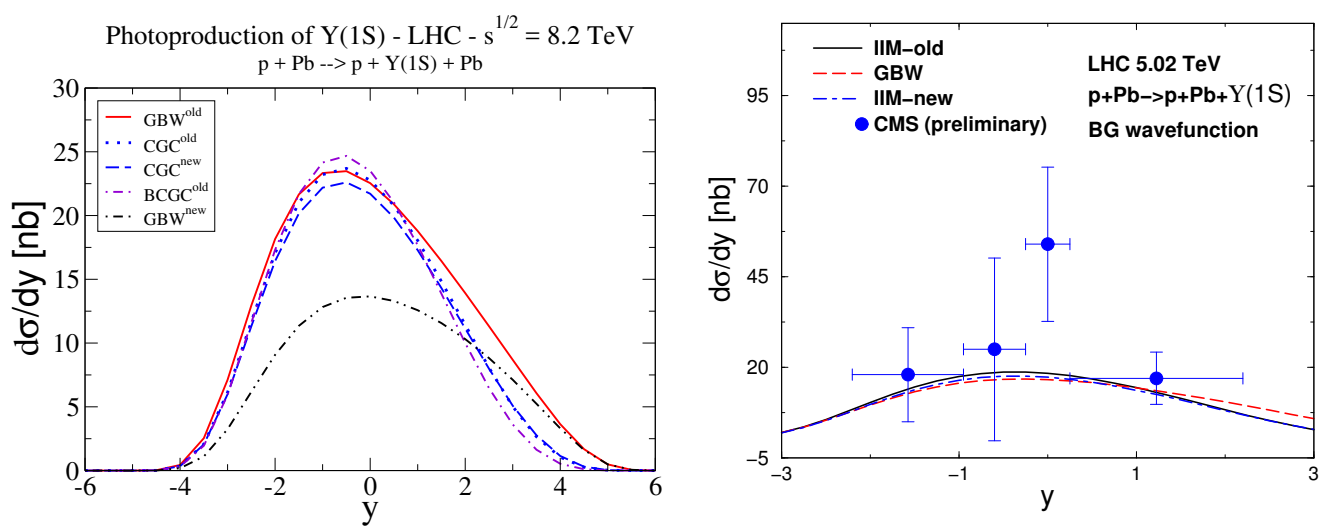

Figure 2. Rapidity distribution for the vector meson state $\Upsilon(1 S)$ in $p A$ collisions at $\sqrt{s}=8.2 \mathrm{TeV}$ (left panel) and at 5.02 TeV (right panel). Data from CMS Collabotation [27].
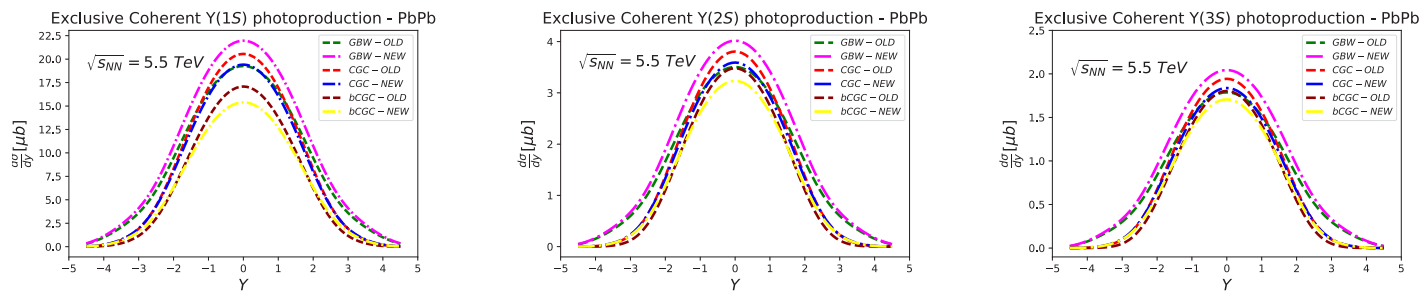

Figure 3. Exclusive coherent photoproduction of $\Upsilon(1 S, 2 S, 3 S)$ in $\mathrm{PbPb}$ at $\sqrt{s}=5.5 \mathrm{TeV}$ for GBW, CGC and bCGC dipole models.

excitations, taking into account the different models presented in the last section. The theoretical uncertainty is relatively large, being of order $15 \%$ for the $1 S$ state (similar for the remaining $2 S$ and $3 S$ states). We could have an additional uncertainty related to the vector meson wave function, however in Ref. [28] it was shown that this is not the case for $\Upsilon$ states (the overall theoretical uncertainty is within the experimental error bars in $p p$ case [8]). For the main contribution, we have $d \sigma_{c o h} / d y(y=0)=18.5 \pm 3.5 \mu \mathrm{b}$ for $\Upsilon(1 S)$. The relative contribution of the excited states compared to the bound states is $\Upsilon(1 S) / \Upsilon(2 S) / \Upsilon(3 S)=1 / 0.17 / 0.09$. We see that the relative normalization and the overall behavior is changed mostly at mid-rapidity when comparing the old and updated versions of the dipole cross sections (the deviation at large rapidities is less evident). Notice that the LHCb data for upsilon production in $p p$ collisions is reproduced by all the models in the forward region [8] as shown in Ref. [7]. Therefore,the current level of the experimental uncertainties does not allow us to make definitive statements about the precision of the models considered. For sake of completeness, we present the integrated cross sections considering distinct cuts on rapidity. In Table 1, we present the results for the full rapidity coverage, $-4<y<4$, and forward rapidities, $2 \leq y \leq 4.5$. In both tables 1 and 2, we present only the updated versions of the dipole cross sections.

We now focus on the incoherent reaction, $P b P b \rightarrow P b \Upsilon P b^{*}$. This is a new contribution to the literature concerning the upsilon production. The rapidity distribution is shown in Fig. 4 using the same notation as the previous figure. As already known, the incoherent cross section is smaller 

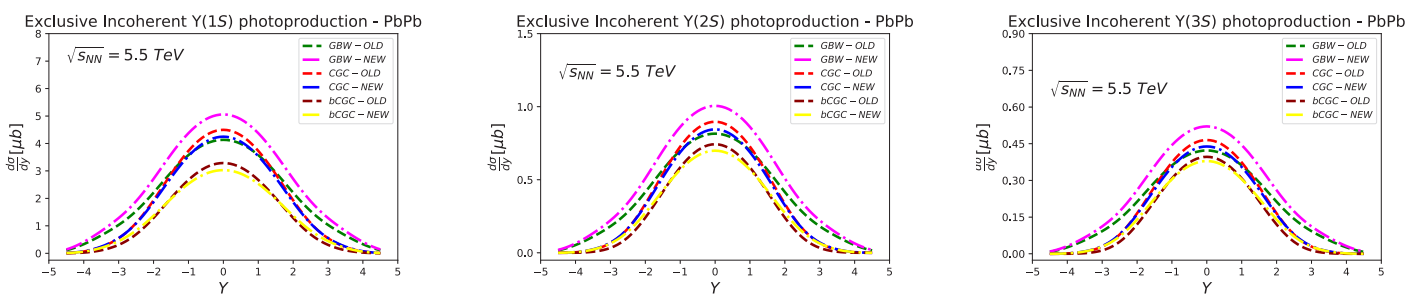

Figure 4. Exclusive incoherent photoproduction of $\Upsilon(1 S, 2 S, 3 S)$ in $\mathrm{PbPb}$ at $\sqrt{s}=5.5 \mathrm{TeV}$ for GBW, CGC and bCGC dipole models.

Table 1. Integrated cross section (in units of $\mu \mathrm{b}$ ) for coherent reactions, $P b P b \rightarrow P b \Upsilon P b$, for full rapidity coverage (and forward rapidities). Here, we consider the updated versions of dipole cross sections.

\begin{tabular}{cccc}
\hline process: $\mathrm{PbPb}$ & $\sqrt{s}=5.5 \mathrm{TeV}$ & $|y| \leq 4(2 \leq y \leq 4.5)$ & \\
$\Upsilon(n S)$ & $\mathrm{GBW}$ & $\mathrm{CGC}$ & $\mathrm{b}-\mathrm{CGC}$ \\
$\Upsilon(1 S)$ & $163.7(60.8)$ & $171.9(63.8)$ & $143(53.1)$ \\
$\Upsilon(2 S)$ & $20.3(7.8)$ & $22.0(8.2)$ & $20.5(7.7)$ \\
$\Upsilon(3 S)$ & $10.3(3.9)$ & $11.9(4.3)$ & $10.9(4.1)$ \\
\hline
\end{tabular}

Table 2. Integrated cross section (in units of $\mu$ b) for incoherent reactions, $P b P b \rightarrow P b \Upsilon P b^{*}$, for full rapidity coverage (and forward rapidities).Here, we consider the updated versions of dipole cross sections.

\begin{tabular}{cccc}
\hline \hline process: $\mathrm{PbPb}$ & $\sqrt{s}=5.5 \mathrm{TeV}$ & $|y| \leq 4(2 \leq y \leq 4.5)$ & \\
$\Upsilon(n S)$ & $\mathrm{GBW}$ & $\mathrm{CGC}$ & $\mathrm{b}-\mathrm{CGC}$ \\
$\Upsilon(1 S)$ & $61.2(25.6)$ & $58.5(24.4)$ & $44.5(18.6)$ \\
$\Upsilon(2 S)$ & $8.2(3.4)$ & $8.0(3.4)$ & $6.9(2.9)$ \\
$\Upsilon(3 S)$ & $4.2(1.8)$ & $4.2(1.8)$ & $3.8(1.6)$ \\
\hline
\end{tabular}

than the coherent one. The typical ratio is $\left(d \sigma_{\text {inc }} / d y\right) /\left(d \sigma_{c o h} / d y\right) \simeq 0.2$. For instance, we obtain $d \sigma_{\text {inc }} / d y(y=0)=3.75 \pm 1.25 \mu \mathrm{b}$ for $\Upsilon(1 S)$. The theoretical uncertainty seems to be larger than in the coherent case. The integrated cross sections are shown in Table 2 in the rapidity ranges $|y| \leq 4$ and $2 \leq y \leq 4.5$.

The calculations performed above can be compared to another theoretical approaches available in the literature. Let start comparing them to the STARlight Monte Carlo [14]. For the coherent production, the predictions for the $\Upsilon$ state ratios are lower that STARlight results. As discussed in Ref. [7], the possible origin comes from the extrapolation of HERA-DATA and taking a fixed ratio for the distinct states in the Monte Carlo, whereas in current case the evolution on energy is dynamically generated by the parton saturation approach models and mostly by the meson wavefuntions for the radially excited states. The nuclear effects are also computed in a different way in the two formalisms. In STARLight, the nuclear shadowing is calculated using vector meson dominance (VDM) plus Glauber model for hadronic collisions. In our case, shadowing comes from the multiple scatterings of color dipoles and is described by the Glauber-Gribov approach. We verified that our results are also smaller that in Ref. [14], which can be related to more shadowing in color dipole models compared to the VDM+Glauber approach. In Ref. [30] only the coherent contribution was computed and 
the theoretical uncertainty we have found in the color dipole approach is comparable to perturbative QCD formalism. Concerning similar dipole calculations, more recent investigations are available in Refs. [28, 31]. In Ref. [31] only the coherent $\Upsilon(1 S)$ production has been considered at $5.02 \mathrm{TeV}$. The results are smaller than ours and the main reason is the wavefunction chosen (Light Cone Gaussian wavefunction which gives smaller overall normalization compared to Boosted Gaussian one). The authors in [31] did not investigated the theoretical uncertainty associated to the wavefunction and dipole cross sections as well (only the uncertainty coming from one model for dipole cross section was addressed). In Ref. [28] the theoretical uncertainty for the coherent and incoherent cross section was investigated. However, predictions for higher energies in $\mathrm{PbPb}$ collisions were not presented and only the $\Upsilon(1 S)$ state was considered (the results are consistent with ours in that case). Finally, we did not consider photonuclear breakup in the present study. We will consider them in future analysis as they are important and the distinct channels have been measured for $\rho$ and $J / \psi$ photoproduction in UPCs $[32,33]$. This sort of analysis was recently done in Ref.[34], where the coherent $\Upsilon(1 S)$ production was considered using a pQCD model with NLO accuracy. An important point discussed in [34] is that the large $y$ region gives the dominant contribution for $0 \mathrm{nXn}$ and $\mathrm{XnXn}$ channel and they probe larger photon-target centre-of-mass energy than the case without neutron tagging.

\section{Summary}

We presented the predictions of rapidity distribution and integrated cross sections for the $\Upsilon(1 S, 2 S, 3 S)$ states for the LHC energies, including discussions for $p p$ and $p A$ modes and fosusing mainly on $\mathrm{PbPb}$ collisions. The rapidity intervals used in total cross section were selected to match with the rapidity coverage of LHCb and ALICE detectors both covering $2 \leq y \leq 4.5$. The main contribution is the computation of the incoherent cross section within the color dipole approach and Glauber-Gribov treatment of nuclear shadowing. The cross section for the excited states are also calculated in a consistent formalism where the wavefunction of $2 S$ and $3 S$ states are theoretically well constrained. The usual procedure in the literature involves only an extrapolation of DESY-HERA production ratios to the LHC energies. Our calculations are directly comparable to the STARLight calculation, where distinct procedures are involved in the computation of nuclear shadowing (VDM plus Glauber model versus color dipole plus Glauber-Gribov approach) and how to obtain the incoherent cross section. The color dipole model is in agreement with measurements of $\Upsilon(1 S)$ state in $p p$ and $p A$ collisions.

\section{Acknowledgments}

I would like to thank the organizers of XLVII International Symposium on Multiparticle Dynamics (ISMD2017) for warm hospitality and for an exciting conference.

\section{References}

[1] G. Baur, K. Hencken, D. Trautmann, S. Sadovsky, Y. Kharlov, Phys. Rep. 364, 359 (2002); C. A. Bertulani, S. R. Klein and J. Nystrand, Ann. Rev. Nucl. Part. Sci. 55, 271 (2005).

[2] N. N. Nikolaev, B. G. Zakharov, Phys. Lett. B 332, 184 (1994); Z. Phys. C 64, 631 (1994).

[3] J. Nemchik, N. N. Nikolaev, E. Predazzi and B. G. Zakharov, Phys. Lett. B 374, 199 (1996).

[4] F. Gelis, E. Iancu, J. Jalilian-Marian and R. Venugopalan, Ann. Rev. Nucl. Part. Sci. 60, 463 (2010); H. Weigert, Prog. Part. Nucl. Phys. 55, 461 (2005); J. Jalilian-Marian and Y. V. Kovchegov, Prog. Part. Nucl. Phys. 56, 104 (2006); A.L. Ayala, M.B. Gay Ducati and E.M. Levin, Nucl.Phys. B 493, 305 (1997). 
[5] A. Dainese, in Proceedings of the 38th International Symposium of Multiparticle Dynamics (ISMD2008): Hamburg, Germany. September 15-20 2008, p.118-124, arXiv:0902.0377 [hep-ph].

[6] M. B. Gay Ducati, F. Köpp and M.V. T. Machado. Phys. Rev. D 96, 054001 (2017).

[7] M. B. Gay Ducati, F. Köpp, M.V. T. Machado, and S. Martins. Phys. Rev. D 94, 094023 (2016).

[8] R. Aaij et al. [LHCb Collaboration], JHEP 1509, 084 (2015).

[9] K. Dusling , F. Gelis, T. Lappi and R. Venugopalan, Nucl. Phys. A 836, 159 (2010) .

[10] T. Lappi, H. Mäntysaari, R. Venugopalan, Phys. Rev. Lett. 114, 082301 (2015). H. Mäntysaari and B. Schenke, Phys. Rev. Lett. 117, 052301 (2016).

[11] L. Frankfurt, G.A. Miller and M. Strikman, Phys. Rev. Lett. 71, 2859 (1993); A. Caldwell and H. Kowalski, Phys. Rev. C 81, 025203 (2010).

[12] Kirill Tuchin, Phys. Rev. C 79, 055206 (2009); T. Lappi and H. Mäntysaari, Phys. Rev. C 83 065202 (2011) ; T. Toll and T. Ullrich, Phys. Rev. C 87, 024913 (2013) ; H. Mäntysaari, B. Schenke, C. Shen and P. Tribedy, Phys. Lett. B 772, 681 (2017).

[13] J. Cepila, J.G. Contreras, J. D. Tapia Takaki, Phys. Lett. B 766, 186 (2017).

[14] S.R. Klein, J. Nystrand, J. Seger, Y. Gorbunov and J. Butterworth, Comput. Phys. Commun. 212, 258 (2017).

[15] S. Klein and J. Nystrand, Phys. Rev. C 60, 014903 (1999).

[16] B. Z. Kopeliovich and B. G. Zakharov, Phys. Rev. D 44, 3466 (1991); Yu.P.Ivanov, J.Huefner,

B.Z.Kopeliovich and A.V.Tarasov, AIP Conf.Proc. 660, 283 (2003).

[17] J. Nemchik, N. N. Nikolaev, E. Predazzi and B. G. Zakharov, Z. Phys. C 75, 71 (1997).

[18] B.E. Cox, J. R. Forshaw and R. Sandapen, JHEP 0906 , 034 (2009).

[19] K. Golec-Biernat and M. Wüsthoff, Phys. Rev. D 59, 014017 (1998).

[20] E. Iancu, K. Itakura, S. Munier, Phys. Lett. B 590, 199 (2004).

[21] H. Kowalski, L. Motyka and G. Watt, Phys. Rev. D 74, 074016 (2006).

[22] G. Soyez, Phys. Lett. B 655, 32 (2007).

[23] A.H. Rezaeain and I. Schmidt, Phys. Rev. D 88, 074016 (2013).

[24] W. Schäfer, A. Szczurek, Phys. Rev. D 76, 094014 (2007); A. Rybarska, W. Schäfer, A. Szczurek, Phys. Lett. B 668 , 126 (2008).

[25] S.P. Jones, A.D. Martin, M.G. Ryskin and T. Teubner, J. Phys. G: Nucl. Part. Phys. 43, 035002 (2016).

[26] V.P Gonçalves, B.D. Moreira and F.S. Navarra, Phys. Lett. B 742, 172 (2015); V.P Gonçalves,

B.D. Moreira, F.S. Navarra and D. Spiering, Phys. Rev. D 94, 014009 (2016); G. Gil da Silveira,

V.P Gonçalves and M.M. Jaime, Phys.Rev. D 95034020 (2017).

[27] R. Chudasama et al. [CMS Collaboration], PoS ICPAQGP 2015, 042 (2017), [arXiv:1607.00786 [hep-ex]].

[28] G. Sampaio dos Santos and M.V.T. Machado, J. Phys. G 42, 105001 (2015).

[29] N . Armesto,A. H. Rezaeian, Phys.Rev. D 90, 054003 (2014).

[30] A. Adeluyi and A. Nguyen, Phys. Rev. C 87, 027901 (2013).

[31] V.P. Gonçalves, B.D. Moreira and F.S. Navarra, Phys. Rev. D 95, 054011 (2017).

[32] J. Adam et al. [ALICE Collaboration], JHEP 1509, 095 (2015).

[33] V. Khachatryan et al. [CMS Collaboration], Phys. Lett. B 396, 772 (2017).

[34] V. Guzey, E. Kryshen and M. Zhalov, Phys. Rev. C 93, 055206 (2016). 